\title{
A Map for Understanding Decision Making
}

\author{
Michael J. O'Brien, Professor of Anthropology and Dean, College of Arts and \\ Science, University of Missouri
}

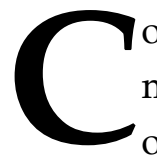

ontributions to this year's Merrill Research Retreat focus on analytics - those metrics that one might use to estimate a university's excellence relative to that of its peers in various key areas. My paper builds on these studies and others like them, but my purpose is a bit different in that I focus on how people use such information to make decisions, especially when they are faced with an ever-increasing quantity and array of information, regardless of source or kind. I am, for example, just as interested in how and why people choose a particular brand of shampoo in their local Walmart as I am in how they select which academic journals to read and which research projects to pursue.

The world today is a blur compared to what it was even a hundred years ago. Humans evolved in a world of few but significant choices, whereas most of us now live in a consumer world of almost countless, interchangeable ones, whether we're shopping for shampoo or deciding what to read. Digital media now record almost all of these choices, and these "digital shadows" are increasingly becoming the subjects of "big data" research. Some see this trend as a boon to understanding human behavior because of the sheer size of data sets that result from modern technology, including such things as cell phones and the Internet, but there are caveats. Before we delve too deeply into endless piles of information, it wouldn't hurt to have at least a casual understanding of how humans process information, especially in the era of big data. The brief overview I present below might be useful for university administrators if for no other reason than, say, it provides a starting point for understanding how faculty members, especially those in the sciences and behavioral sciences, navigate through the onslaught of research-related information they face on a continuous basis. Not only has there been an exponential growth in scientific articles over the last decade, the annual growth in the number of journals is likewise staggering. How does one make good decisions-meaning those that are in the best interests (long as well as short term) of the researcher - when faced with an information overload?

Several years ago, some of my colleagues and I began to review what has been written on the subject of decision making. What we found was that it had become commonplace for those involved with information processing to casually dip into the social sciences to see what tools they could borrow to better understand human behavior. That's fine to a point, but one problem with these free- 
wheeling forays is that the various social sciences make considerably different assumptions about the behaviors they describe. To the classical economist, for example, rational actors monopolize the human stage, each with a predictable ability to maximize benefits and minimize costs. These self-contained individuals rarely depend on anyone but themselves for learning new behaviors. By contrast, evolutionary psychologists as- learning from prestigious people in the kinship group, making alliances, and so forth. Indeed, evolutionary anthropologists and psychologists have argued persuasively that the anomalously large brain (neocortex) size in humans evolved primarily for social-learning purposes. ${ }^{1}$

In view of the different processes and scales involved in decision making, especially decisions about the quality of a behavior or product, how do we deter-

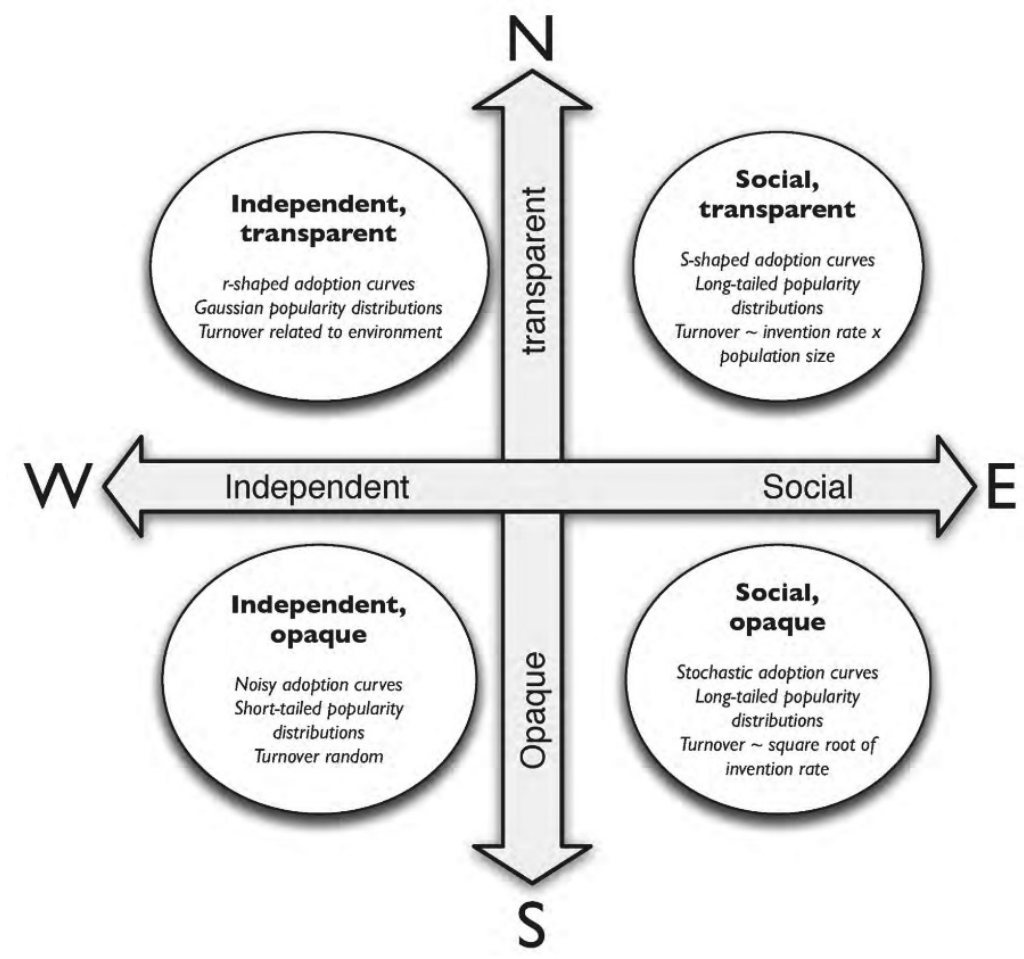

Figure 1. A conceptual map for understanding human behavior that plots case studies on two axes. ${ }^{7}$ The horizontal axis represents how agents make decisions. At the western end, agents learn individually, whereas at the eastern end they base decisions solely on the choices of others-they copy. The vertical axis represents the transparency of options in terms of payoffs and risks, from total transparency at the northern end to complete opaqueness at the southern end. The characteristics in the bubbles are intended to convey likely possibilities, not certitudes.

sume choices are driven by ancient hunter-gatherer instincts that can seem irrational in a modern western society. Anthropologists have yet different assumptions, namely that humans are highly social animals. This means that almost all their decisions involve social learning and negotiation-sharing food, mine which one predominates in a given situation? And what about the different perspectives that the disciplines bring? Is one right and the others wrong? No. The different disciplines operate at different scales, and they all make different, but useful, assumptions, ranging from the 
psychology of the thoughtful, isolated individual to the sociology of frantic market populations. At one extreme, an individual makes a well- (or otherwise-) informed decision based on careful analysis, and at the other extreme, people effectively copy one another without thinking about it.

In several recent publications, my colleagues and I show how big data - the kind most businesses and government bodies already possess - can be used to "map" decisions along two dimensions: social influence and information (Figure 1). ${ }^{2-7}$ Granted, it is a simple heuristic map-and I do little more than summarize it here-but it captures the essential elements of human decision making that should be of concern to businesses, marketers, and even university administrators. As we demonstrate, the data often show that "I'll have what she's having" is a better default setting than "I'll select the rational option."

The Map The north-south axis represents how well people are informed about their decisions. At the northern edge of the map are behaviors that have some immediate, detectable, and consistent impact of getting a decision right or wrong. The key word here is "detectable," which means that an agent clearly sees and understands the landscape of costs and benefits associated with a decision. At the southern edge are decisions where there is no measurable difference in benefits, often where people are poorly informed about their choices or otherwise overwhelmed by "decision fatigue."

The east-west axis represents the degree to which agents make their decisions individually or socially. At the far west is one hundred percent individual learning, where agents rely only on their own knowledge of the costs and benefits of a particular behavior. At the far eastern edge is pure social learning, where people do only as others do.

The map requires a few simplifying assumptions to keep it from turning into something so large that it loses its usefulness for generating potentially fruitful research hypotheses. First, it treats the various competencies of agents-intelligence, education, cognitive skills, and so on - as real but too fine-grained to be visible at the scale of data aggregated across a population and/or time. Second, agents are not assumed to know what is best for them in terms of long-term satisfaction, fitness, or survival, given that rational agents, who are very good at sampling the environment, are not omniscient. Third, the distinction is blurred between learning and decision making. Technically, they are separate actions, but this distinction draws too fine a line around what ultimately influences an agent's decision and how clearly the agent can distinguish among potential payoffs. Fourth, although the map represents a continuous space, it is divided into quadrants for ease of discussion and application to example datasets. Any characterizations are based on extreme positions of agents within each quadrant. As agents move away from extremes, the characterizations are relaxed.

Not surprisingly, at any given moment populations are mixtures of social learners and individual learners. Every individual makes some decisions on his or her own and spends part of the time saying, "I'll have what she's having." Equally unsurprising is the fact that the balance between social and individual 
learning is important to how communities behave. This has been realized in studies of fish schools, bird flocks, and animal herds, where experiments reveal, for example, that logical, coordinated behavior of an entire school can result from a majority who are copying their neighbors and a small minority who are acting individually, such as swimming toward a physical target in the pool. The school of fish might look as if all fish know where they are going when in fact only a very few are so well informed, but their swimming direction diffuses through the school by means of social learning. A naive observer might see a school of goaldirected individuals, which would plot in the northwest, when in fact the school plots toward the southeast-mostly poorly informed social learning interspersed with informed individual action. It doesn't take much of an imagination to extend these examples to academic settings, including large research groups.

Why might any of this matter? Because most policymaking assumes that people all reside in the northwest-people make their own decisions asocially, with their own goals and preferences. Although we might recognize types of behavior-for example, the modal behavior of assistant professors in a research-intensive physics departmentwe would, nonetheless, look at specific decisions as made by rational agents. To marketers, the northwest captures the implicit assumption embedded in surveys about a product, regardless of social context-the friends and influences surrounding the product. Both behavioral economics, with its (slightly) imperfect actors and their cognitive quirks, as well as evolutionary psychology, with its supposedly evolved preferences, go in the northwest quadrant.

The map would be just another fourbox heuristic if it were not for the fact that it relates specifically to patterns we can resolve from behavioral data, whether those data come from sales records or citations to scholarly articles and books. In terms of sales, the data would ideally pertain to the relative popularity of all available options through time, but more practically, it works just as well with a list of the most popular choices through time, such as weekly bestseller lists, for example. It works the same with respect to scientific citations, showing what (and who) is hot and what's not.

\section{A Quick Tour Around the Map}

Perhaps a brief tour around the map will make this clearer. We can start in the northwest, as this is where the vast majority of economics has been for over a century. The northwest is where disseminated information about a new service or product is enough - the medical literature for a beneficial new pharmaceutical product, for example - because each individual has the time, motivation, and knowledge to think through all the inherent costs and benefits of the decision. Laptop screen size, for instance, plots in the northwest because it shows a diagnostic bell curve of popularity, which reflects an optimal size that fits most people's needs. This means that people can choose individually based on clear physical constraints. The same applies to academic disciplines, where (we might suppose) researchers continuously update their information about which topics are hot and which ones are not. Bell curvesthe signature of the northwest-center on 
the best cost-benefit option and change little until something better comes along.

More and more, though, people are forced to make rapid decisions from among a dizzying array of options and may resort to guesswork. This leads us toward the southwest, where people make poorly informed choices on their own. A novice choosing from among hundreds of listed mutual funds, or looking at a wall of similar eyeglasses, may just as well pick at random. With huge selections and not much difference among them, cheap commercial products often lie in the southwest-too similar for anyone to notice in any social context. Hopefully, few of us in academic professions get caught in the southwest-certainly not an attractive place to be if one's goal is tenure and promotion.

With a great many options, the popularity of any particular choice is essentially a lottery. Half a century ago, marketing scientist Andrew Ehrenberg laid out the analytical expectations for the southwest quadrant. ${ }^{8}$ He showed that when consumers cannot tell the difference, the distribution of brand popularity is "short-tailed," meaning that the probability of an option becoming extremely popular-the tail of the distributionfalls off exponentially. Also, when people resort to guesswork, there should be no consistency in the rank-order of popularity from one time period to the next. So while laptop screen size lies in the northwest, most of the different laptop models-hundreds and hundreds of black laptops out there-plot in the southwest.

Contrary to our default assumptions, it turns out that most behavioral questions of interest do not, at least according to market data, actually plot in the northwest or southwest but rather in the eastern half of the map. Our intuition says we make our own decisions, but the data say that we are almost constantly influenced by other people's decisions. Ideally, we are informed about our social influences-we listen to experts or we copy the most successful or prestigious people on a particular topic. Copying the most successful individuals means we have enough information to recognize real talent, such as hunter-gatherers knowing who the best hunter is in their small band or a group of graduate students knowing who the best scientist is in a biology department. Copying better results is social learning we all understand, and it explains why the bow and arrow rapidly spread throughout eastern North America 1400 years ago and why hybrid corn spread across the American Midwest.

In the case of hybrid corn, consider the cumulative percentage of farmers in two Iowa communities who adopted hybrid seed corn over a period of 15 years: It took nine years for the frequency of hybrid planters to reach $20 \%$ but only six more years for it to reach fixation at $99 \%$ (Figure 2). Here we have a classic S-curve, with slow adoption followed by a significant upward shift in 1933-1934 and a peak in 1936-1937. Early on, a few farmers experimented with hybrid corn, but this yielded almost no shifts in behavior until enough farmers began experimenting with it that it finally reached a point where social learning took over. The same pattern can hold in academia, where "hot" new areas are slow to develop but quick to spread once enough researchers are exposed to them. 


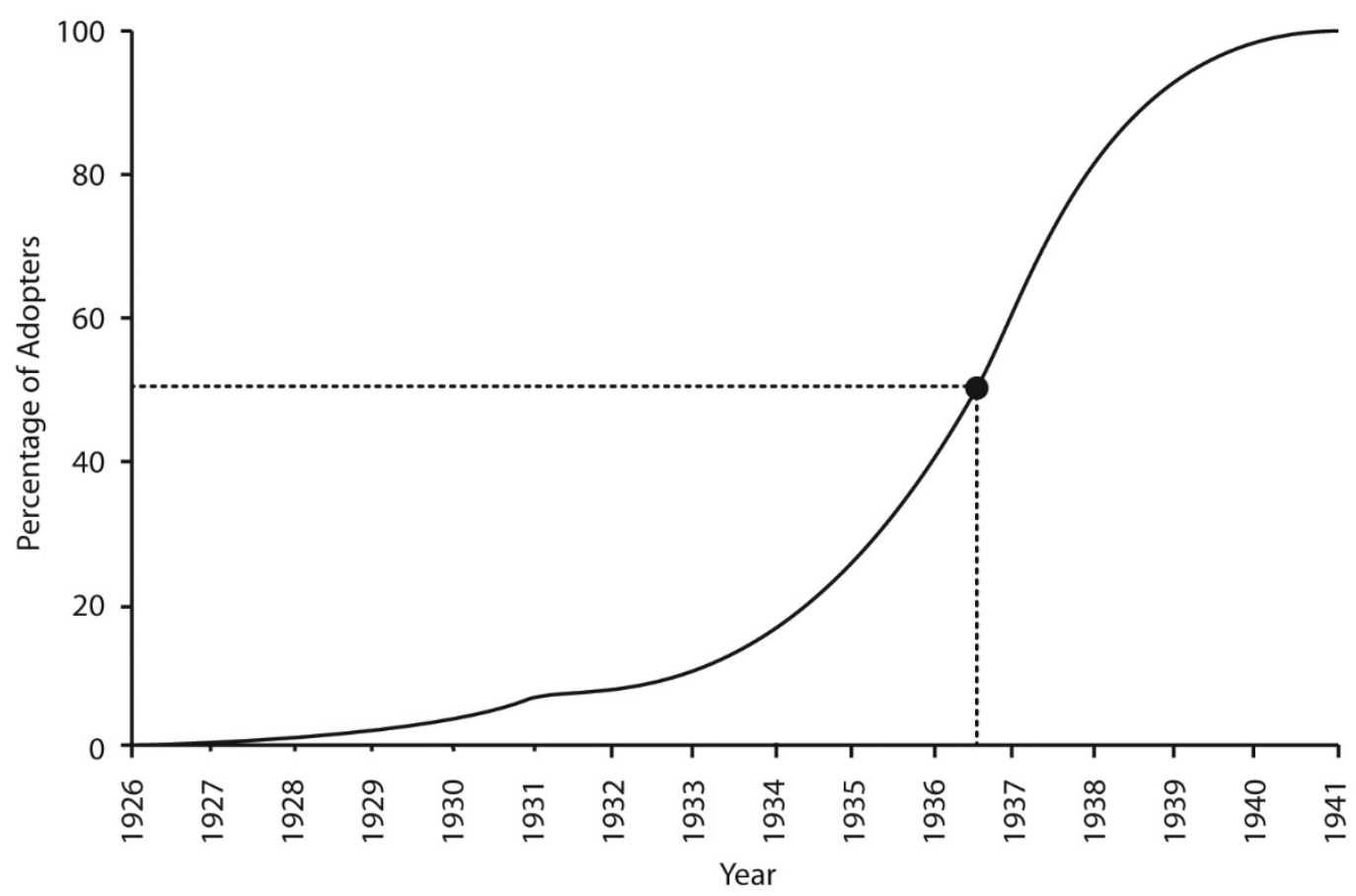

Figure 2. Diffusion curve showing the cumulative use by year of hybrid corn in two Iowa farming communities, 1926-1941.13,14 This diffusion curve is a prototypical example of a "long-tailed" S-curve. The dotted lines mark the point on the curve with the highest rate of change.

Social-learning biases add an extra layer of complexity to social-diffusion models. An agent, for example, might direct attention toward agents who meet one or more of the following criteria: They are prestigious, are related to the agent, are attractive, are similar in behavior to the agent, and so on. Among these copying biases, perhaps the most adaptive for the copier are those that are directed toward an agent or group of agents with which the copier seeks to identify. This can be a type of conformist bias, which can lead to social diffusion within the limits of the group that is conforming. A popular name might diffuse through a generation, a certain dialect through an ethnic community, or a certain set of interlinked customs through a community. In these cases, the copying is directed according to the rule of "copy the majority." True conformity, in the sense of determining the majority decision and copying it, can introduce punctuated effects or a degree of unpredictability that is uncharacteristic of standard diffusion curves. If copying is directed, then larger populations mean that agents often can observe popularity only locally (leaving aside modern online search engines and popularity lists). In other words, agents often try to conform locally rather than globally. When conformity is directed locally, it might mean agents adopt something only after enough of their friends or colleagues have adopted it.

Old as they may be, traditions are never static. All traditions are dynamic by definition, because they are passed down the generations by social learning. We take it for granted that an entire language is re-created in the first years of every person's life, but each time we 
learn a tradition, there are errors or deliberate creative changes. It is remarkable that cultural traditions can endure for so long. In Europe, the Little Red Riding Hood folktale, nuclear families, and square houses are all thousands of years old. Among our ancient hominin ancestors, the same technique for knapping hand axes was taught from one generation to the next for over forty thousand generations. This resilience of human behavior over the generations reflects our remarkably sophisticated ability to learn from one another.

In the northeast quadrant, informed social learning - copying skill, quality, or prestigious individuals-calls on traditional social-diffusion theory, grounded in the model that marketing scientist Frank Bass proposed in 1969. ${ }^{9}$ Because the copying is well informed, there is coherence and logic to group behavior, as with schools of fish. We expect quality to be brought to the fore in the northeast through social interactions, as innovations are discovered and communicated through a population. Better things inevitably become commonplace, but they take time to diffuse smoothly through the relevant population because people must introduce them to one another.

Hierarchical social networks-each of us copying from the ranks above us, for example-help insure that the best options will spread, as shown by Erez Lieberman and colleagues at Harvard University. ${ }^{10}$ The Harvard group found, however, that diffused networks-people copying more indiscriminately - tend to minimize this advantage. When everyone just shrugs and says, "I'll have what she's having," certain things become popular, but there is no guarantee that the best things are what rise to the top. This is the southeast quadrant.

Undirected copying yields continuous turnover in what is most popular, as long as there is some small flux of novel invention in the system. The diagnostic patterns of the southeast-long tail, continual turnover, and stochastic changecan be tested against popularity data. The reason people use deodorant at all-for hygiene-lies in the northwest quadrant, but the market for the brand of deodorant lies in the southeast quadrant. Different brands of the same product are usually in the southeast, especially as people are flooded not only with product choices but also with myriad social influences of recommendations, top-10 lists, and "most popular" search results. Lacking any inherent distinctiveness or any obvious social reputation is how things wind up in the southeast - many brand names, hackneyed clichés, and getting tattoos, for example.

\section{The Age of "What She's Having"}

Understanding the southeast quadrant helps us explain why markets are changing faster than ever and in less predictable ways. Unpredictability is inherent to the southeast. In a controlled experiment, Columbia University's Matt Salganik and colleagues found that people consistently chose the same sorts of music when acting in isolation-northwest - but when they were allowed to see what songs others were downloading, the behavior became more like "I'll have what she's having" - southeast-and the results unpredictable. ${ }^{11}$

Of course, the social version of this already-classic experiment represents the online world today. The 1960's term "future shock" nicely describes our anxiety 
as the world shifts from the northeast, when ancient traditions changed slowly over generations, to the southeast, where indiscriminant copying, random events, and global connectivity spread changes on the daily scale. We evolved in a world of few but important choices, but we live in a world of many, largely interchangeable ones. Just as we feel adapted to the new order of the world, new fashions and technologies wash over us, new buzzwords enter our conversation, and "Buy! Buy!" becomes "Sell! Sell!"

Eric Beinhocker describes the rapidity with which this explosion of diversity has occurred as a hundred-million-fold, or eight orders of magnitude, difference since the time of our hunter-gatherer ancestors a little over 10,000 years ago. ${ }^{12}$ Think about it: There are now over 50,000 restaurants in the greater New York City area and over 200 television channels on cable TV. A Walmart store near JFK International Airport has over 100,000 different items in stock. Talk about choices!

Despite being overwhelmed with meaningless choices and social influences, individual choice is still the marketers' default setting and, in our broader culture, perhaps even something of a religion. This certainly applies to the academic world as well. Canny marketers can use this mistaken assumption to their advantage. If a brand becomes popular in the southeast, through indiscriminant copying, this luck can be consolidated by moving it to the northwest and concocting post-hoc reasons for its success. Or it can be moved to the northeast because of reputation and brand loyalty. Much of what marketers mistakenly call "loyalty" however, remains in the southeast, sustained merely through its own inertia and bound to be ephemeral. Sales data become crucial here, in the patterns distinguishing the southeast from the northeast.

In traditional societies, differences between groups arose over many generations. In northern Cameroon and Chad, for example, neighboring Moussey and Massa groups intermarry and share a common genealogical origin and technology, yet they grow different crops, raise livestock in different ways, and dislike each other's cuisine. Small amounts of randomness-introduced through creativity, nonconformity, or accident-get amplified through local social learning. In this Internet era of decision fatigue, as we are forced to copy more and more, differences between groups may therefore be amplified, despite the "globalized" connectivity.

These elements-flux, learning, selection, and random events - bring about a new age of models of human behavior. If the market no longer fits in the northwest, there is little value in trying to predict rational and optimal outcomes. If the market plots in the southeast, it is better approached as a matter of insurance or secure investment-coping with unpredictability by maximizing probabilities, minimizing risks, and placing many small bets. Probability distributions, population size, invention rate, interaction networks, and time span become the key parameters in floating with the tides. Marketing becomes less about satisfying "the" archetypal consumer and more about how many interconnected consumers affect each other's behavior. Old ideas, such as the sanctity of the "brand," have to be recast in terms of this bigger, more anthropological map. To do all this, 
it pays to have data analysts schooled in evolutionary theory, but if lacking all this, just point to someone and say, "I'll have what she's having." It's almost always a safe bet.

\section{Acknowledgments}

This paper borrows heavily from several articles and a book that several of my colleagues and I have produced over the past several years (referenced in the paper). In particular, I thank William A. Brock, Mark Earls, Paul Ormerod, Philip Garnett, and especially R. Alexander Bentley. My contributions to what truly is a group effort pale in comparison to theirs. I also thank Mabel Rice for her kind invitation to be a part of the 2013 Merrill Conference and Evelyn Haaheim for making the experience so enjoyable.

\section{References}

1. Dunbar RIM. 1993. Coevolution of neocortical size, group size and language in humans. Behavioral and Brain Sciences 16:681-735.

2. Bentley RA, O'Brien MJ, Ormerod P. 2011. Quality versus mere popularity: a conceptual map for understanding human behavior. Mind \& Society 10:181-191.

3. Bentley RA, Earls M, O'Brien MJ. 2011. I'll have what she's having: mapping social behavior. MIT Press, Cambridge, MA.

4. Bentley RA, O’Brien MJ. 2011. The selectivity of social learning and the tempo of cultural evolution. Journal of Evolutionary Psychology 9:125141.

5. O'Brien MJ, Bentley RA. 2011. Stimulated variation and cascades: two processes in the evolution of complex technological systems. Journal of Archaeological Method and Theory 18:309-335.

6. Bentley RA, Garnet P, O'Brien MJ, Brock, WA. 2012. Word diffusion and climate science. PLoS ONE 7(11):e47966.

7. Bentley RA, O'Brien MJ, Brock WA. 2013. Mapping collective behavior in the big-data era. Behavioral and Brain Sciences (in press).

8. Ehrenberg ASC. 1959 The pattern of consumer purchases. Journal of the Royal Statistical Society C 8:26-41.

9. Bass FM. 1969. A new product growth model for consumer durables. Management Science 15:215227.

10. Lieberman E, Hauert C, Nowak MA. 2005. Evolutionary dynamics on graphs. Nature 433:312-316.

11. Salganik MJ, Dodds PS, Watts DJ. 2006. Experimental study of inequality and unpredictability in an artificial cultural market. Science 311:854856.

12. Beinhocker ED. 2006. The origin of wealth: evolution, complexity, and the radical remaking of economics. Random House, New York.

13. Ryan B, Gross NC. 1943. The diffusion of hybrid seed corn in two Iowa communities. Rural Sociology 8:1524.

14. Henrich J. 2001. Cultural transmission and the diffusion of innovations: adoption dynamics indicate that biased cultural transmission is the predominate force in behavioral change. American Anthropologist 103:992-1013. 